\title{
Boundary Controllability and Observability of a One-Dimensional Nonuniform SCOLE System ${ }^{1}$
}

\author{
B. Z. GuO ${ }^{2}$ AND S. A. Ivanov ${ }^{3}$
}

Communicated by F. L. Chernousko

\begin{abstract}
A hybrid system, composed of an elastic beam governed by an Euler-Bernoulli beam equation with variable coefficients and a linked rigid body governed by an ordinary differential equation, is considered. Various controllability/observability properties of the system under bounday control/observation are studied. It is shown that an open-loop smooth/singular controller of either torque control or force control is sufficient to make the system exactly controllable in arbitrarily short time duration.
\end{abstract}

Key Words. Hybrid systems, beam equations, controllability, observability, exponential families, Riesz basis.

\section{Introduction}

Among the variety of large-scale flexible space structures, a prototype model is the NASA spacecraft control laboratory experiment (SCOLE, Ref. 1). The SCOLE model consists of a long flexible mast $\mathrm{M}$, described by a partial differential equation, clamped at one end to a massive spaceship $\mathrm{S}$ at rest after completing some space maneuver and fastened at the other end to a rigid antenna A, governed by an ordinary differential equation, upon which the control is imposed by means of gas jet. For the SCOLE model that is uniform, the exact controllability of the system

\footnotetext{
${ }^{1}$ The work was carried out with the support of the National Natural Science Foundation of China and the Russian Foundation for Fundamental Researches, Grant 02-01-00554.

${ }^{2}$ Professor, Institute of Systems Science, Academy of Mathematics and System Sciences, Academia Sinica, Beijing PRC, and School of Computational and Applied Mathematics, University of the Witwatersrand, Johannesburg, South Africa.

${ }^{3}$ Associate Professor, Russian Center of Laser Physics, St.Petersburg State University, St.Petersburg, Russia.
} 
in the smooth state space was studied first in Ref. 2 based on the theory of semi-infinite beams. The existence of a smooth open-loop controller of both force and torque is proved by a constructive cutoff approach. The exact controllability for the nonuniform SCOLE model in the smooth state space was developed in Ref. 3 via the Riesz basis approach. In the usual energy state space, the exact controllability of the uniform SCOLE model imposing simultaneously both smooth force control and singular torque control was obtained in Ref. 4 by virtue of the HUM (Hilbert uniqueness method), based on the existence and continuity of the weak solution of the hybrid system. Under the limitation of the beam length, the same result was presented also in Ref. 4 for singular torque control. On the other hand, the output feedback stabilization for both the uniform and nonuniform SCOLE models in the smooth state space have been developed in Refs. 5, 6 and Ref. 3, respectively.

In this paper, we study further the controllability and observability properties of the SCOLE model. The contributions of this paper are: (a) our model is nonuniform, hence different from the uniform models for which the same problems are considered in th literature; (b) we obtain a complete description of the reachable set and observability properties for force or torque control in the smooth space or energy state space; (c) the limitation on the beam length, assumed in Ref. 4 for one control in deriving the exact controllability in the energy state space, is removed; (d) the limitation for control time is removed.

The moment approach (Ref. 7) is adopted in this investigation. Torque control case is explained in details in Section 2. The case of force control is discussed briefly in Section 3, since there is no essential technical difference for the two cases. The main idea of the apporach can be explained as follows. The dynamic system is written in the form

$$
\dot{Y}=\mathcal{A} Y+B u,
$$

with a scalar control $u$. The approach reduces the controllability problem to the study of the geometrical properties of the exponential family

$$
\mathcal{E}=\left\{B^{*} \Phi_{n} e^{\lambda_{n} t}\right\},
$$

where $\Phi_{n}$ are the eigenfunctions of the system operator $\mathcal{A}$ corresponding to the eigenvalues $\lambda_{n}$ and $B^{*}$ is the adjoint of the control operator $B$. The asymptotic expansions for $\lambda_{n}$ and $\Phi_{n}$ developed in Ref. 3 allow us to obtain a complete description of the controllability and observability of the system. Concretely, based on the asymptotic behaviour of the eigenpairs of $\mathcal{A}$, it is shown that, for any time $T>0$, the family $\mathcal{E}$ is minimal and forms a Riesz basis for its span in $H^{1}(0, T)$, but an unconditional 
basis (rather than a Riesz basis) for its span in $L^{2}(0, T)$. This leads to the spectral controllability in the energy space and to the exact controllability in the smoother space $D(\mathcal{A})$, the graph space of $\mathcal{A}$. Moreover, if $u$ is the control with minimal energy ( $L^{2}$ norm) that steers the zero initial state to $\Phi$ at time $T$, then the map $u \rightarrow \Phi$ is an isomorphism form $L^{2}(0, T)$ onto $D(\mathcal{A})$.

The controllability of the system using the singular control $u \in$ $H^{-1}(0, T)$ is also discussed. It is shown that, when the singular torque control is imposed, the exact controllability in the energy space can be formulated formally, but the solution to the system is usually not continuous in time, which is contrary to the claim of Ref. 4. However, the dual problem of the observation in $H^{1}(0, T)$ space is found to be well-posed and we obtain the exact observability of the system with singular control in the energy state space. A similar problem exists for the force control case, but in this case the exact observability is valid only in the space $H^{1 / 2}(0, T)$.

1.1. System Operator. Consider the following nonuniform SCOLE model with boundary control:

$$
\begin{aligned}
& \rho(x) y_{t t}(x, t)+\left(E I(x) y_{x x}(x, t)\right)_{x x}=0, \quad 0<x<1, t>0, \\
& y(0, t)=y_{x}(0, t)=0, \\
& m y_{t t}(1, t)-\left(E I y_{x x}\right)_{x}(1, t)=0, \\
& J y_{x t t}(1, t)+E I(1) y_{x x}(1, t)=v(t),
\end{aligned}
$$

where $x$ stands for the position and $t$ for the time, $E I(x)$ is the flexural rigidity of the beam, $\rho(x)$ the mass density at $x, m$ is the mass of the antenna A, and $J$ the moment of inertia of A about its centroid, the point of attachment of the mast $\mathrm{M}, v(t)$ is the control torque applied to $\mathrm{A}$. We refer to Refs. 2 and 5 for the necessary physical background of the model. In what follows, we assume always that

$$
\rho(x), E I(x) \in C^{4}[0,1], \quad E I, \rho, m, J>0 .
$$

The natural energy state space for the hybrid system (1) is the Hilbert space

$$
H=H_{E}^{2}(0,1) \oplus L^{2}(0,1) \oplus C^{2}, \quad H_{E}^{2}(0,1)=\left\{f \in H^{2}(0,1) \mid f(0)=f^{\prime}(0)=0\right\},
$$

in which the inner product induced norm is defined by

$$
\|(f, g, \xi, \eta)\|^{2}=\int_{0}^{1}\left[E I(x)\left|f^{\prime \prime}(x)\right|^{2}+\rho(x)|g(x)|^{2}\right] d x+m^{-1}|\xi|^{2}+J^{-1}|\eta|^{2},
$$


for any $(f, g, \xi, \eta) \in H$. Define the system operator $\mathcal{A}: H \supset D(\mathcal{A}) \rightarrow H$ as follows:

$$
\begin{aligned}
& \mathcal{A}(f, g, \xi, \eta)=\left[g,-(1 / \rho(x))\left(E I(x) f^{\prime \prime}(x)\right)^{\prime \prime},\left(E I f^{\prime \prime}\right)^{\prime}(1),-E I(1) f^{\prime \prime}(1)\right] \\
& D(\mathcal{A})=\left\{(f, g, \xi, \eta) \in\left(H_{E}^{2} \cap H^{4}\right) \oplus H_{E}^{2} \oplus C^{2}, \xi=m g(1), \eta=J g^{\prime}(1)\right\}
\end{aligned}
$$

With the operator $\mathcal{A}$ at hand, we can write equations (1) as

$$
\begin{aligned}
& (d / d t) Y(t)=\mathcal{A} Y(t)+B v(t), \quad B=(0,0,0,1), \\
& Y(t)=\left(y(\cdot, t), y_{t}(\cdot, t), m y_{t}(1, t), J y_{x t}(1, t)\right) .
\end{aligned}
$$

The following propositions come from Ref. 3.

\section{Proposition 1.1.}

(i) The operator $\mathcal{A}$ is skew-adjoint and its spectrum consists of only simple eigenvalues $\left\{\lambda_{n}\right\}$.

(ii) By almost normalized eigenfunctions $\Phi_{n}$ corresponding to $\lambda_{n}$, that we represent as $\Phi_{n} \asymp 1$, we mean that there are constants $c_{1}, c_{2}$ independent of $n$ such that $0<c_{1} \leq\left\|\Phi_{n}\right\| \leq c_{2}$.

(iii) $\Phi_{n}$ takes the form

$\Phi_{n}=\left(\lambda_{n}^{-1} \phi_{n}, \phi_{n}, m \phi_{n}(1), J \phi_{n}^{\prime}(1)\right)$,

where $\phi_{n} \neq 0$ satisfies

$\lambda_{n}^{2} \rho(x) \phi_{n}(x)+\left(E I(x) \phi_{n}^{\prime \prime}(x)\right)^{\prime \prime}=0, \quad 0<x<1$,

$\phi_{n}(0)=\phi_{n}^{\prime}(0)=0$,

$m \lambda_{n}^{2} \phi_{n}(1)-\left(E I \phi_{n}^{\prime \prime}\right)^{\prime}(1)=0$,

$J \lambda_{n}^{2} \phi_{n}^{\prime}(1)+E I(1) \phi_{n}^{\prime \prime}(1)=0$.

Proposition 1.2. The following asymptotic formulas are valid:

$\lambda_{n}=i(n+1 / 2)^{2} \pi^{2} \operatorname{sign}(n) / h^{2}+O(1)$, 


$$
\begin{aligned}
e^{p(x)} \phi_{n}(x)= & \sin (n+\pi / 2) z-\cos (n+\pi / 2) z \\
& +e^{-(n+1 / 2) \pi z}-(-1)^{n} e^{-(n+1 / 2) \pi(1-z)}+\mathcal{O}\left(n^{-1}\right),
\end{aligned}
$$

where

$$
\begin{aligned}
& p(x)=(1 / 4) \int_{0}^{z(x)} a(\tau) d \tau, \\
& z=(1 / h) \int_{0}^{x}[\rho(\tau) / E I(\tau)]^{1 / 4} d \tau, \quad h=\int_{0}^{1}[\rho(\tau) / E I(\tau)]^{1 / 4} d \tau \\
& a(z)=(3 h / 2)[\rho(x) / E I(x)]^{-5 / 4}(d / d x)[\rho(x) / E I(x)] \\
& +h\left[2 E I^{\prime}(x) / E I(x)\right](\rho(x) / E I(x))^{-1 / 4} \text {. } \\
& \left|\phi_{n}^{\prime}(1)\right| \asymp 1 / n^{2}, \quad n \in Z, n \neq 0 .
\end{aligned}
$$

\section{Control/Observation Formulation}

Because the system (4) is a linear time-invertible system, the general controllability problem is equivalent to the reachability problem of the following system with zero intial state;

$$
Y(t)=\mathcal{A} Y(t)+B v(t), \quad Y(0)=0
$$

where $B$ is an operator from the real line to the state space. We consider the torque control only in this section. In this case, $B$ is defined as

$$
B \alpha=(0,0,0, \alpha), \quad \forall \alpha \in \mathbb{R}
$$

The state space is taken to be either the energy space $H$ or $D(\mathcal{A})$, depending on the context.

The dual observation problem is

$$
\begin{aligned}
& \dot{Z}=\mathcal{A} Z, \quad Z(0)=Z_{0}, \\
& w(t)=B^{*} Z(t)=Z_{4}(t),
\end{aligned}
$$


where

$B^{*}: H \ni \Phi \rightarrow B^{*} \Phi=(\Phi)_{4} \in \mathbb{C}$

and $(\Phi)_{4}$ denotes the last component of the vector $\Phi \in \mathbb{R}^{4}$.

By reachable set $R(T)$ at time $T>0$, we mean the set of all states $Y^{*}$ in the state space such that there is at least one control $v$ such that the solution to (10) satisfies $Y(T)=Y^{*}$.

\section{Definition 2.1.}

(i) The system (10) is called spectrally controllable on $[0, T]$ for the time $T>0$ if the reachable set $R(T)$ contains all the eigenmodes $\Phi_{n}$.

(ii) The system (10) is called approximately controllable on $[0, T]$ for the time $T>0$ if the reachable set $R(T)$ is dense in the state space.

(iii) The system (10) is called exactly controllable on $[0, T]$ for the time $T>0$ if the reachable set $R(T)$ coincides with the state space.

\section{Definition 2.2.}

(i) The system (11) is called approximately observable on $[0, T]$ for the time $T>0$ if the knowledge of the output $w(t)$ on $[0, T]$ determines the initial state $Z_{0}$ uniquely.

(ii) The system (11) is called exactly observable on $[0, T]$ for the time $T>0$ if the initial state $Z_{0}$ can be uniquely and continuously constructed from the knowledge of the output $w(t)$ on $[0, T]$.

2.1. Exponential Family. In order to study the controllability/observabibility problems via the moment approach, we need to investigate the geometric properties of the associated exponential family. Set

$$
\mathcal{E}_{0}:=\left\{e^{\lambda_{n} t}\right\}_{n \in Z}
$$

Introduce the upper density $D$ for a real sequence $\mathcal{M}=\left\{\mu_{n}\right\}$,

$$
D:=\lim _{r \rightarrow \infty} \sup _{x \in \mathbb{R}}[n(x+r)-n(x)] / r
$$

where $n(x)$ is the number of $\mu_{n}$ in $[0, x]$ if $x \geq 0$ or $[x, 0]$ if $x<0$.

Since $i \lambda_{n} \in \mathbb{R}$ and $\left|\lambda_{n}\right| \asymp n^{2}$, it follows that $n(x) \asymp \sqrt{|x|}$; hence, $D=0$ when $\mathcal{M}=\left\{i \lambda_{n}\right\}$. 
Proposition 2.1. See Ref. 8. Let $\mathcal{M}=\left\{\mu_{n}\right\} \subset \mathbb{R}$. If the set $\mathcal{M}$ is separable, i.e., $\inf _{m \neq n}\left|\mu_{n}-\mu_{m}\right|>0$ and $D(\mathcal{M})<T / 2 \pi$, then there exists a set $\Xi=\left\{\xi_{n}\right\}$ such that the family

$$
\mathcal{E}_{M} \cup \mathcal{E}_{\Xi}, \quad \mathcal{E}_{\Xi}:=\left\{e^{\xi_{n} t}\right\}, \quad \mathcal{E}_{M}:=\left\{e^{\mu_{n} t}\right\}
$$

forms a Riesz basis for $L^{2}(0, T)$.

Definition 2.3. A Riesz basis for its span is called an $\mathcal{L}$-basis.

Corollary 2.1. For any $T>0$, the family $\mathcal{E}_{0}$ forms an $\mathcal{L}$-basis for $L^{2}(0, T)$.

Proof. For any given $T>0$, it follows from Proposition 2.1 that there exists a family $\mathcal{E}_{\Xi}$ such that $\mathcal{E}_{0} \cup \mathcal{E}_{\Xi}$ forms a Riesz basis for $L^{2}(0, T)$. The result then follows from the fact that part of the Riesz basis is an $\mathcal{L}$-basis too.

Let

$$
\Theta=\left\{\theta_{n}(t)\right\}
$$

be the family biorthogonal to $\mathcal{E}_{0}$ in $L^{2}(0, T)$. Since the family of exponentials $\left\{e^{\lambda_{n} t}\right\}$ is not complete in $L^{2}(0, T)$, the biorthogonal family is not unique. Indeed, if $\left\{\theta_{n}\right\}$ is a biorthogonal family and if $\tilde{\theta}_{n}-\theta_{n} \perp \mathcal{E}$ for all $n$, then evidently $\left\{\theta_{n}\right\}$ also forms a biorthogonal family. In what follows, we consider the biorthogonal family of $\mathcal{E}_{0}$ in $\overline{\operatorname{span} \mathcal{E}_{0}}$. Note that this choice is such that the norms of $\theta_{n}$ of this family are minimal. It can be seen also that the biorthogonal family to $\left\{q_{n} e^{\lambda_{n} t}\right\}$, where $q_{n}$ are scalars, is $\left\{\theta_{n}(t) / q_{n}\right\}$.

Theorem 2.1. For any $T>0$ and any $s>0, \mathcal{E}_{0}$ forms an unconditional basis for its span in the Sobolev space $H^{s}(0, T)$.

In order to prove Theorem 2.1, we need the following result.

Proposition 2.2. See Ref. 9. Suppose that the family $\mathcal{E}_{M}:=\left\{e^{\mu_{n} t}\right\}_{n \in \mathrm{N}}$ forms a Riesz basis for $L^{2}(0, T)$ for some time $T>0$. Then, for any $m \in$ $\mathrm{N}$ and any number of different points $\xi_{1}, \xi_{2}, \ldots, \xi_{m}$ satisfying $\mu_{n} \neq \xi_{m}$ for all $n, m \in \mathrm{N}$, the family $\mathcal{E}_{M} \cup\left\{e^{e i \xi_{n} t}\right\}_{n=1}^{m}$ forms an unconditional basis for $H^{m}(0, T)$.

Proof of Theorem 2.1. The proof for the case of integer $m>0$ can be obtained easily from Proposition 2.2. Indeed, since there exists a set $\Xi$ 
such that the joint family $\mathcal{E}_{0} \cup\left\{e^{\xi_{n} t}\right\}$ forms a Riesz basis for $L^{2}(0, T)$, it follows from Proposition 2.2 that $\mathcal{E}_{0}$ forms an unconditional basis for its span in $H^{m}(0, T)$ as part of an unconditional basis.

Next, we give a sketch of the proof for the case of noninteger $m$. Without loss of generality, we limit ourselves to the case $0<s<1$ and $T=1$. Take the reference family

$$
\tilde{\Xi}=\left\{\tilde{\xi}_{n}\right\}, \tilde{\xi}_{n}=n-(q / 2) \operatorname{sign}(n),
$$

with

$$
q \neq s-1 / 2 \text { and }|q|<1 / 4 .
$$

Then (see e.g. Ref. 10 ), the family $\mathcal{E}_{\Xi}$ forms an unconditional basis for $H^{p}(0,1)$ for any $p<1 / 2-q$ and unconditional basis for its span in $H^{p}(0,1)$ for $p>1 / 2-q$.

For any $\lambda_{n}$, take the closest point $\xi_{m_{n}}$ of $\tilde{\Xi}$. If there are two closest points, we take any one of them. Now, we remove $\tilde{\xi}_{m_{n}}$ from $\tilde{\Xi}$ and denote the remaining set as $\hat{\Xi}$. We compare $\tilde{\Xi}$ and $\hat{\Xi}$. The difference is only that the point $\tilde{\xi}_{m_{n}}$ is replaced by $\lambda_{n}$. We see that

$$
m_{n} \asymp n^{2} \quad \text { and } \quad\left|\lambda_{n}-\tilde{\xi}_{m_{n}}\right|<1 .
$$

Lemma 4.5 and Theorem 4.7 of Ref. 10 give that the basis property in $H^{s}$ of the disturbed family $\hat{\Xi}$ coincides with one of $\tilde{\Xi}$. The result follows.

2.2. $\boldsymbol{L}^{2}$-Control/Observation in Energy Space. In this section, we consider the reachability problem for the system (10) in the energy space $H$ : for the given final state $\Phi \in H$ and time $T>0$, find the control $v \in$ $L^{2}(0, T)$ such that

$$
Y(T)=\Phi .
$$

Since the eigenfunctions $\left\{\Phi_{n}\right\}_{n \in Z}$ form a Riesz basis for $H$, we can write the solution of (10) and $\Phi$ as

$$
Y(t)=\sum_{n \in \mathbf{Z}} c_{n}(t) \Phi_{n}, \quad \Phi=\sum_{n \in \mathbf{Z}} c_{n}^{0} \Phi_{n} .
$$

The control term $B v(t)$ is represented also in terms of $\left\{\Phi_{n}\right\}$ as follows:

$$
B v(t)=v(t) B 1=v(t) \sum_{n \in \mathbf{Z}} b_{n} \Phi_{n},
$$




$$
b_{n}=\left(B 1, \Phi_{n}\right) /\left\|\Phi_{n}\right\|^{2}=\left(\Phi_{n}\right)_{4}=J \phi_{n}^{\prime}(1) /\left\|\Phi_{n}\right\|^{2} .
$$

By (10), we have

$$
\dot{c}_{n}(t) \Phi_{n}=\sum_{n \in \mathbf{Z}} \lambda_{n} c_{n}(t) \Phi_{n}+\sum_{n \in \mathbf{Z}} v(t) b_{n} \Phi_{n} .
$$

Since $Y(0)=0$, the coefficients $c_{n}(t)$ satisfy the following first-order ordinary differential equations:

$$
\dot{c}_{n}(t)=\lambda_{n} c_{n}(t)+b_{n}, \quad c_{n}(0)=0, \quad \forall n \in Z .
$$

Therefore,

$$
c_{n}(T)=\int_{0}^{T} b_{n} e^{\lambda_{n}(T-t)} v(t) d t .
$$

Set

$$
\mathcal{E}=\left\{b_{n} e^{\lambda_{n} t}\right\}, \quad \tilde{v}=\bar{v}(T-t) .
$$

We have the following moment problem with respect to $\mathcal{E}$ :

$$
c_{n}(T)=\left(b_{n} e^{\lambda_{n} t}, \tilde{v}\right) L^{2}(0, T)
$$

It is seen that the reachable set $R(T)$ is isomorphic to the set of all sequences $\left\{c_{n}(T)\right\} \in \ell^{2}$ such that the moment problem (14) possesses a solution $v \in L^{2}(0, T)$. This means that there exists a solution to (12) if and only if, for any sequence $\left\{c_{n}(T)\right\} \in \ell^{2}$, there exists a control $v \in L^{2}(0, T)$ that solves the moment problem (14). A general discussion on the moment approach can be found in Ref. 7 .

Let us introduce the control operator

$$
W: L^{2}(0, T) \ni v \rightarrow Y(T)=\sum_{n \in \mathbf{Z}}\left(b_{n} e^{\lambda_{n} t}, \tilde{v}\right) \Phi_{n} \in H .
$$

As usual, the observation operator $\mathcal{O}$ is defined as the adjoint of $W$,

$$
H \ni Z_{0}=\sum_{n \in \mathbf{Z}} z_{n} \Phi_{n} \rightarrow w(t)=B^{*} Z(t)=Z_{4}(t)=\sum_{n \in \mathbf{Z}} z_{n} b_{n} e^{\lambda_{n} t} \in L^{2}(0, T) .
$$

It is well known (see e.g. Definitions 4.1.3. and 4.1.12 of Ref. 11) that (i) the system (10) is exactly [resp. approximately] controllable on $[0, T]$ in $H$ if and only if $W$ is an isomorphism [resp. injection] from the control space onto the state space and that (ii) the system (11) is exactly [resp. approximately] observable on $[0, T]$ in $H$ if and only if $\mathcal{O}$ is an isomorphism [resp. injection] from the state space to the control space. 
Theorem 2.2. For any $T>0$, let the state space be $H$ and let the control $v \in L^{2}(0, T)$. Then, the system (10) is spectrally but not exactly controllable on $[0, T]$.

Proof. The result on the exact controllability is a consequence of general results for infinite-dimensional systems with finite-rank control operator; see e.g. Theorem 4.1.5 of Ref. 11. Here, we give a simple proof of this claim by the moment approach. Indeed, the system is exactly controllable if and only if the family $\mathcal{E}$ forms an $\mathcal{L}$-basis for $H$. Now, it is seen that the family $\mathcal{E}$ differs from the $\mathcal{L}$-basis family $\mathcal{E}_{0}$ by the factors

$$
b_{n}=J \phi_{n}^{\prime}(1) /\left\|\Phi_{n}\right\|^{2} .
$$

In view of (9),

$$
\left|b_{n}\right| \asymp|n|^{-2},
$$

for sufficiently large integers $|n|$. Together with the fact that $b_{n} \neq 0$, due to $\phi_{n}^{\prime}(1) \neq 0$, this claims that the family $\mathcal{E}$ is not almost normalized,

$$
\left\|b_{n} e^{\lambda_{n} t}\right\| \rightarrow 0, \quad \text { as }|n| \rightarrow \infty .
$$

Hence, $\mathcal{E}$ is not an $\mathcal{L}$-basis for $H$, proving the claim.

By the moment approach, the spectral controllability is equivalent to the minimality of the family $\mathcal{E}$. Because the target state now is $\Phi_{m}$, the corresponding moment problem becomes

$$
\left(b_{n} e^{\lambda_{n} t}, \tilde{v}\right)=0, \quad \text { for } n \neq m, \quad\left(b_{m} e^{\lambda_{m} t}, \tilde{v}\right)=1 .
$$

Since $\mathcal{E}_{0}$ is an $\mathcal{L}$-basis for $L^{2}(0, T)$ for any $T>0$, it follows that $\mathcal{E}$ is minimal. The family that is biorthogonal to $\mathcal{E}$ is nothing but $\left\{\theta_{n}(t) / b_{n}\right\}$. Thus, the control $\tilde{v}$ that solves the moment problem (17) can be taken as

$$
\tilde{v}(t)=\theta_{n}(t) / \overline{b_{n}},
$$

where $\overline{b_{n}}$ is the conjugate of $b_{n}$. It is well-known that such a control has minimal $L^{2}$ norm. The proof is complete.

For the dual system, we have the following theorem.

Corollary 2.2. Let $H$ be the state space. For any $T>0$, the system (11) is approximately, but not exactly, observable on $[0, T]$. 
Proof. By Theorem 2.2, the system (10) is spectrally controllable; hence, it is approximately controllable because the reachable set contains all the eigenmodes. Furthermore, since $\mathcal{A}^{*}=-\mathcal{A}$, it follows from the wellknown duality principle on the cotrollability and observability of linear systems (see e.g. Ref. 11, p.157) that the system (11) is approximately observable in the space $H$ on $[0, T]$ for any $T>0$. The exact observability of the system (11) follows from similar arguments.

We indicate that Corollary 2.2 can be explained simply via the moment approach by showing that the observation operator $\mathcal{O}$ is invertible, but its inverse is not bounded. Let us observe the operator $\mathcal{O}$ acting on the eigenmodes. Let

$$
Z(0)=Z_{0}=\Phi_{n}
$$

Then, the solution of (11) is

$$
\begin{aligned}
& Z(t)=e^{\lambda_{n} t} \Phi_{n}, \\
& B^{*} Z(t)=J \phi_{n}^{\prime}(1) e^{\lambda_{n} t} .
\end{aligned}
$$

Hence,

$$
\Phi_{n} \| \asymp|n|^{-2} .
$$

Finally we indicate that, for the system (10) in the state space $H$, the control $v=v_{n} \in L^{2}(0, T)$ of minimal $L^{2}$ norm that steers the rest initial state to $\Phi_{n}$ has the estimates

$$
C^{-1}(T)|n|^{2} \leq\left\|v_{n}\right\|_{L^{2}(0, T)} \leq C(T)|n|^{2}, \quad \text { for all } n \in \mathbf{Z},
$$

where the constant $C(T)$ depends only on $T$. Indeed, from (17),

$$
\tilde{v}_{n}(t)=\theta_{n}(t) / \bar{b}_{n}
$$

Since the biorthogonal system $\Theta$ forms a Riesz basis for $\overline{\text { span } \mathcal{E}_{0}}$, the norms of the elements of $\Theta$ are uniformly bounded with respect to $n$. Therefore,

$$
\left\|v_{n}\right\|_{L^{2}(0, T)} \asymp\left|b_{n}\right| .
$$

(19) then follows from (9). 
2.3. $L^{2}$-Control/Observation in Smooth Space. From the discussions of the last section, we see that the reason that the control operator is not an isomorphism from the control space onto the energy space is attributed to the fact that the map from the control space to the state space is not balanced in the sense that the control space $L^{2}$ is too small and the state space $\mathrm{H}$ is too large. To balance the situation, we may expand the control space $L^{2}$ or reduce the state space $H$. Some efforts have been made in Ref. 4 with additional conditions on the length of the beam.

From the previous section, we see that the solution $Y(T)$ is smoother than an arbitrary element of $H$ in the sense of $Y(T) \in D(\mathcal{A})$, specifically,

$$
\begin{aligned}
\mathcal{A} Y(T) & =\mathcal{A} \sum_{n \in Z} b_{n}\left(e^{\lambda_{n} t}, \tilde{v}\right)_{L^{2}(0, T)} \Phi_{n} \\
& =\sum_{n \in Z} b_{n} \lambda_{n}\left(e^{\lambda_{n} t}, \tilde{v}\right)_{L^{2}(0, T)} \Phi_{n} .
\end{aligned}
$$

Since $\left|b_{n} \lambda_{n}\right| \asymp 1$ and $\mathcal{E}_{0}$ is a $\mathcal{L}$-basis for $L^{2}(0, T)$, it follows that $\left\{b_{n} \lambda_{n}\left(e^{\lambda_{n} t}, \tilde{v}\right)_{L^{2}(0, T)}\right\} \in \ell^{2}$. Moreover the control sequence operator $W$ acts $L^{2}(0, T)$ onto $\mathrm{D}(\mathcal{A})$, which is valid because that $\mathcal{E}_{0}$ is a $\mathcal{L}$-basis for $L^{2}(0, T)$; the following moment problem:

$$
b_{n} \lambda_{n}\left(e^{\lambda_{n} t}, \tilde{v}\right)_{L^{2}(0, T)}=c_{n}
$$

has a solution $\tilde{v} \in L^{2}(0, T)$ for any sequence $\left\{c_{n}\right\} \in \ell^{2}$. This suggests that a natural state space for $L^{2}$ control is $D(\mathcal{A})$. Let us discuss this in a slightly more general setting.

Set

$$
|\mathcal{A}|:=\sqrt{-\mathcal{A A}^{*}},
$$

which is a self-adjoint operator with the eigenvalues $\left|\lambda_{n}\right|$ and the eigenfunctions $\left\{\Phi_{n}\right\}$. Define

$$
\mathcal{H}^{s}:=D\left(|\mathcal{A}|^{s}\right)=\left\{\left.f \sum_{n \in \mathbf{Z}} f_{n} \Phi_{n}\left|\sum_{n \in \mathbf{Z}}\right| \lambda_{n}\right|^{2 s}\left|f_{n}\right|^{2} \mid<\infty\right\}, \quad s \geq 0 .
$$

It is easy to check that $\left\{\left|\lambda_{n}\right|^{-s} \Phi_{n}\right\}$ is a Riesz basis for $\mathcal{H}^{s}$. Roughly speaking,

$$
\mathcal{H}^{s}=\mathrm{H}^{4 s} \oplus \mathrm{H}^{2 s} \oplus C^{2}
$$

upon the boundary conditions. In particular, $\mathcal{H}^{1}=D(\mathcal{A})$. 
Control in Smooth Space. Consider the control system (10) in the state space $\mathcal{H}^{s}$. Again, we find the solution of (10) in terms of the Riesz basis $\left|\lambda_{n}\right|^{-s} \Phi_{n}$,

$$
Y(t)=\sum_{n \in \mathbf{Z}} c_{n}(t)\left|\lambda_{n}\right|^{-s} \Phi_{n} \in \mathcal{H}^{s},
$$

where $c_{n}(t)$ satisfies

$$
\dot{c}_{n}(t)=\lambda_{n} c_{n}(t)+b_{n}\left|\lambda_{n}\right|^{s} v(t), \quad c_{n}(0)=0 .
$$

Therefore,

$$
c_{n}(T)=\int_{0}^{T} b_{n}\left|\lambda_{n}\right|^{s} e^{\lambda_{n}(T-t)} v(t) d t .
$$

Set

$$
\mathcal{E}_{1}=\left\{b_{n}\left|\lambda_{n}\right|^{s} e^{\lambda_{n} t}\right\}
$$

We have the following moment problem with respect to $\mathcal{E}_{1}$ :

$$
c_{n}(T)=\left(b_{n}\left|\lambda_{n}\right|^{s} e^{\lambda_{n} t}, \tilde{v}\right)_{L^{2}(0, T)} .
$$

By virtue of (9),

$$
\left|b_{n} \| \lambda_{n}\right|^{s} \asymp\left|\lambda_{n}\right|^{s-1} .
$$

If we take $s=1$, then the family $\left\{b_{n}\left|\lambda_{n}\right|^{s} e^{\lambda_{n} t}\right\}$ is almost normalized. Since it is an unconditional basis for $L^{2}(0, T)$, it follows that $\left\{b_{n}\left|\lambda_{n}\right|^{s} e^{\lambda_{n} t}\right\}$ forms a Riesz basis for $L^{2}(0, T)$. This leads to the exact controllability of the system $(10)$ in $\mathcal{H}^{1}=D(\mathcal{A})$ on $[0, \mathrm{~T}]$ for any $\mathrm{T}>0$. This result removes the limitation of $\mathrm{T}$ for the result of the exact controllability obtained in Ref. 3 via the Riesz basis approach and the Ingham inequality.

Now, the control operator for the space $\mathcal{H}^{1}$ is

$$
W_{1}: L^{2}(0, T) \ni v \longrightarrow Y(T)=\sum_{n \in \mathbf{Z}}\left(b_{n}\left|\lambda_{n}\right| e^{\lambda_{n} t}, \tilde{v}\right) \Phi_{n} \in \mathcal{H}^{1}
$$

and the observation operator $\mathcal{O}_{1}=W_{1}^{*}$ is

$$
\mathcal{O}_{1}: \mathcal{H}^{1} \ni Z_{0} \longrightarrow w(t)=B^{*} Z_{0}(t)=\sum_{n \in \mathbf{Z}} z_{n} b_{n}\left|\lambda_{n}\right| e^{\lambda_{n} t} \in L^{2}(0, T) .
$$

In view of the Riesz basis property,

$$
\|W v\|_{\mathcal{H}^{1}} \asymp\|v\|_{L^{2}(0, T)}, \quad\left\|O Z_{0}\right\|_{L^{2}(0, T)} \asymp\left\|Z_{0}\right\|_{\mathcal{H}^{1}} .
$$

Thus, we have proved the following theorem. 
Theorem 2.3. For any time $\mathrm{T}>0$ :

(i) The system (10) with control $v \in L^{2}(0, T)$ is exactly controllable on $[0, T]$ in $\mathcal{H}^{1}$.

(ii) The system (11) is exactly observable on $[0, T]$ in $\mathcal{H}^{1}$ with the observation

$$
B^{*} Z(t)=Z_{4}(t) \in L^{2}(0, T) .
$$

Observation in Smooth Space. The observation operator introduced in (16) has the form

$$
\mathcal{O} Z_{0}=\sum_{n \in \mathbf{Z}} z_{n} b_{n} e^{\lambda_{n} t}
$$

Since $b_{n}$ is decreasing, the inverse of $\mathcal{O}$ is not bounded in $L^{2}(0, T)$. On the other hand, since the exponential family $\left\{b_{n} e^{\lambda_{n} t}\right\}$ forms an unconditional basis for any Sobolev space $H^{s}(0, T), s \geq 0$ and $\left\|b_{n} e^{\lambda_{n} t}\right\|_{H^{1}(0, T)} \asymp 1$, it follows that $\left\{b_{n} e^{\lambda_{n} t}\right\}$ forms an $\mathcal{L}$-basis for $H^{1}(0, T)$. Therefore [compare with (21)]

$$
\left\|\mathcal{O} Z_{0}\right\|_{H^{1}(0, T)} \asymp\left\|Z_{0}\right\|_{H},
$$

which implies that $\mathcal{O}: H^{1} \longrightarrow H$ is bounded invertible in $H^{1}(0, T)$. Thus, we have the following observability result.

Theorem 2.4. For any $T>0$, the system (11) with the observation $B^{*} Z(t)=Z_{4}(t) \in H^{1}(0, T)$ is exactly observable in $\mathcal{H}^{1}=D(\mathcal{A})$.

Results on Singular Control. We consider the following singular control problem of the system (10) with $v \in\left(H^{1}(0, T)\right)^{\prime}$ :

$$
\dot{Y}=\mathcal{A} Y+B v, \quad Y(0)=0, \quad v \in\left(H^{1}(0, T)\right)^{\prime} .
$$

The control operator now becomes

$$
W:\left(H^{1}(0, T)\right)^{\prime} \ni v \longrightarrow H .
$$

We are still interested in the same exact controllability problem with singular control in the energy space $\mathrm{H}$ as we have done for $L^{2}$ control. The representation (15) is still used by the moment approach in this case.

Now, the inner product $(f, g)_{L^{2}(0, T)}$ is extended to the dual product $\langle f, g\rangle$ for $f \in H^{1}(0, T)$ and $g \in\left(H^{1}(0, T)\right)^{\prime}$. That is to say, $\langle f, g\rangle$ is 
the complex conjugate value of the functional $g$ acting on the element $f$. Thus, we may write the moment problem as follows:

$$
C_{n}(T)=\left\langle b_{n} e^{\lambda_{n} t}, \tilde{v}\right\rangle, \quad v \in\left(H^{1}(0, T)\right)^{\prime} .
$$

From Theorem 2.3, the family $\mathcal{E}=\left\{b_{n} e^{\lambda_{n} t}\right\}$ form a Riesz basis for $H^{1}(0, T)$. So does the biorthogonal family $\Theta$ of $\mathcal{E}$ for $\left(H^{1}(0, T)\right)^{\prime}$. Hence, the formal solution to the moment problem (23) is

$$
\tilde{v}=\sum_{n \in \mathbf{Z}} c_{n} \theta_{n} \in\left(H^{1}(0, T)\right)^{\prime} .
$$

In other words, the moment problem (23) admits always a solution for any $\left\{c_{n}(T)\right\} \in \ell^{2}$.

Thus, if we understand that the solution (22) at $t=T$ is

$$
Y(T)=\sum_{n \in \mathbf{Z}}\left\langle b_{n} e^{\lambda_{n} t}, \tilde{v}\right\rangle \Phi_{n},
$$

then we can say, as for usual $L^{2}$ control problem, that the system (10) is exactly controllable on $[0, T]$ for any $T>0$ in the energy space $H$ with singular control $v \in\left(H_{1}(0, T)\right)^{\prime}$. Unfortunately, the control problem (22) is not well-posed, in the sense that the solution to (22) is not continuous in time, which is contrary to the claim of Ref. 4. For example, if we take

$$
v(t)=\delta(t-T) \in\left(H^{1}(0, T)\right)^{\prime},
$$

the Dirac function, then the solution to (22) is zero before $t=T$ and

$$
Y(T)=\sum_{n \in \mathbf{Z}}\left\langle b_{n} e^{\lambda n t}, \delta(t)\right\rangle \Phi_{n}=\sum_{n \in \mathbf{Z}} b_{n} \Phi_{n}=B 1=(0,0,0,1) .
$$

The same thing takes place for the delta function inside $(0, T), v(t)=$ $\delta\left(t-t_{0}\right)$.

The claim that the solution to (22) is not continuous in time can be justified also by the limit process. Suppose that $\left\{v_{m}\right\} \in L^{2}(0, T)$ is a sequence of smooth functions approaching $v(t)=\delta\left(t-t_{0}\right)$ in $\left(H^{1}(0, T)\right)^{\prime}$,

$$
v_{m}(t) \rightarrow \delta\left(t-t_{0}\right) \in\left(H^{1}(0, T)\right)^{\prime} .
$$

Then, the following moment equalities hold for all $m$ :

$$
C_{n}(t)=\left(b_{n} e^{\lambda_{n} \tau}, \tilde{v}_{m}\right)_{L^{2}(0, t)}=\left\langle b_{n} e^{\lambda_{n} \tau}, \tilde{v}_{n}\right\rangle .
$$


Letting $m \rightarrow \infty$, we obtain

$$
\begin{array}{ll}
c_{n}(t)=0, & \text { as } t<t_{0}, \\
c_{n}\left(v_{m}, t\right)=b_{n}, & \text { as } t>t_{0} .
\end{array}
$$

However, the limit solution $Y(t)$ has a jump $B 1=(0,0,0,1)$ at $t_{0}$. Hence, for singular control, the meaning of exact controllability is not in clear in the sense of usual understanding and needs further investigation.

Remark 2.1. In Ref. 4, the author claimed that the solution to problem (22) is continuous in time in $H$. In the proof of this claim, the author used the following identity:

$$
\left\langle f, g^{\prime}\right\rangle=-\left(f^{\prime}, g\right)_{L^{2}(0, t)},
$$

for all $f \in H^{1}(0, t)$ and $g \in\left(H^{1}(0, t)\right)^{\prime}$. This does not appear to be the classical definition of generalized derivative in the Sobolev space $H^{1}(0, t)$. Moreover, this relation is not true for all $t \in(0, T)$, even for smooth functions $f$ and $g$. In fact, if $f$ and $g^{\prime}$ are both in $L^{2}(0, T)$, then the dual product $\left\langle f, g^{\prime}\right\rangle$ coincides with the inner product $\left(f, g^{\prime}\right)_{L^{2}(0, t)}$.

\section{Force Control/Observation}

In this section, we list the results for the force control case without going to the proof in details, since the proofs can be given along the same line as for the case of torque control. The control problem that we consider in this section is

$$
\dot{Y}=\mathcal{A} Y+B_{u} v, \quad Y(0)=0, \quad B_{v} \alpha=(0,0, \alpha, 0) .
$$

The dual system is

$$
\dot{Z}=\mathcal{A Z}, \quad Z(0)=Z_{0}, \quad w(t)=B_{u}^{*} Z(t)=Z_{3}(t),
$$

where $Z_{3}$ is the third component of the vector $Z \in \mathbb{R}^{4}$.

\section{1. $L^{2}$-Control/Observation in Energy Space. Write}

$$
b_{n}^{u}=\left(B_{u} 1, \Phi_{n}\right) /\left\|\Phi_{n}\right\|^{2}=\left(\Phi_{n}\right)_{3} /\left\|\Phi_{n}\right\|^{2}=m \phi_{n}(1) /\left\|\Phi_{n}\right\|^{2} \text {. }
$$

Then,

$$
B_{u} u(t)=u(t) B_{u} 1=\sum_{n \in \mathbf{Z}} b_{n}^{u} \Phi_{n} .
$$


For any $T>0$, apply the moment approach to obtain the moment equalities

$$
C_{n}(T)=\left(b_{n}^{u} e^{\lambda_{n} t}, \tilde{u}\right)_{L^{2}(0, T)},
$$

with respect to $\mathcal{E}_{u}=\left\{b_{n}^{u} e^{\lambda_{n} t}\right\}$. The only difference between (27) and (14) is that the coefficients $b_{n}$ in (14) are replaced by

$$
b_{n}^{u}=m \phi_{n}(1) /\left\|\Phi_{n}\right\|^{2},
$$

where $\phi_{n}(1)$ has the asymptotic expansion

$$
\left|\phi_{n}(1)\right| \asymp 1 /|n|, \quad n \in Z, \quad n \neq 0 .
$$

(28) does not appear explicitly in Ref. 3, but can be obtained easily after a straightforward computation used there. Also, $\phi_{n}(1)$ is not identically zero for all $n$.

Now, the control is

$$
W_{u}: L^{2}(0, T) \ni u \rightarrow Y(T)=\sum_{n \in \mathbf{Z}}\left(b_{n}^{u} e^{\lambda_{n} t}, \tilde{u}\right) \Phi_{n} \in H
$$

and the observation operator becomes

$$
\mathcal{O}_{u}: H \ni Z_{0} \rightarrow w(t)=B_{u}^{*} Z_{0}(t)=\sum_{n \in \mathbf{Z}} z_{n} b_{n}^{u} e^{\lambda_{n} t} \in L^{2}(0, T),
$$

where

$$
B_{u}^{*}: H \ni \Phi \rightarrow B_{u}^{*} \Phi=(\Phi)_{3} \in \mathbf{C} .
$$

Theorem 3.1. For any $T>0$, the system (25) with the control $u \in$ $L^{2}(0, T)$ and the state space $H$ is spectrally but not exactly controllable on $[0, T]$.

Proof. The proof is similar to that of Theorem 2.2.

For the exact controllability, it is noted that the family $\mathcal{E}_{u}$ differs from the $\mathcal{L}$-basis family $\mathcal{E}_{0}$ by the factors $b_{n}^{u}=m \phi_{n}(1) /\left\|\Phi_{n}\right\|^{2}$. By virtue of (9), $\left|b_{n}^{u}\right| \asymp|n|^{-1}$ for all sufficiently large integers $|n|$. Together with $\phi_{n}(1) \neq 0$, this gives $b_{n} \neq 0$. Hence, the family $\mathcal{E}_{u}$ is not almost normalized,

$$
\left\|b_{n}^{u} e^{\lambda_{n} t}\right\| \rightarrow 0, \quad \text { as }|n| \rightarrow \infty .
$$

Therefore, the system (25) is not exactly controllable on any $[0, T]$ in $H$. 
Next, for the target state $\Phi_{m}$, the corresponding moment problem becomes

$$
\left(b_{n}^{u} e^{\lambda_{n} t}, \tilde{u}\right)=0, \text { as } n \neq m, \quad\left(b_{m} e^{\lambda_{m} t}, \tilde{u}\right)=1 .
$$

Therefore, $\tilde{u}$ can be chosen as the $m$ th element of the biorthogonal family $\mathcal{E}_{u}$. Since the family biorthogonal to $\mathcal{E}_{u}$ is $\left\{\theta_{n}(t) / \overline{b_{n}^{u}}\right\}$, the control of minimal $L^{2}$ norm that steers the system (25) to $\Phi_{n}$ is just $\theta_{n}(t) / \overline{b_{n}^{u}}$. This shows that the system (25) is spectrally controllable on $[0, T]$. 2.2 .

For the dual system, we have the following result parallel to Corollary

Corollary 3.1. For any $T>0$, the system (26), with the observation $B_{u}^{*} Z=Z_{3}(t) \in L^{2}(0, T)$, is approximately but not exactly observable in $H$.

In this case, we also have the estimate for the observation operator on the eigenmodes. In other words, for $Z(0)=\Phi_{n}$, the solution to (26) is

$$
Z(t)=e^{\lambda_{n} t} \Phi_{n}
$$

hence,

$$
B_{u}^{*} Z(t)=n \phi_{n}(1) e^{\lambda_{n} t} .
$$

This yields

$$
\left\|\mathcal{O}_{u} \Phi_{n}\right\| \asymp|n|^{-1} .
$$

3.2. Smooth Observation/Singular Control in Energy Space. By (28),

$$
\left|b_{n}^{u}\right|\left|\lambda_{n}\right|^{s} \asymp\left|\lambda_{n}\right|^{s-1 / 2} .
$$

Take $s=1 / 2$. Then, the family $\left\{b_{n}^{u}\left|\lambda_{n}\right|^{s} e^{\lambda_{n} t}\right\}$ is almost normalized. Since the family $\left\{b_{n}^{u}\left|\lambda_{n}\right|^{S} e^{\lambda_{n} t}\right\}$ is an unconditional basis for $H^{1 / 2}(0, T)$ for any $T>0$ by Theorem 2.3, so it is a Riesz basis for $\mathcal{H}^{1 / 2}(0, T)$. This leads to the exact controllability of the system $(25)$ in $\mathcal{H}^{1 / 2}=D(\sqrt{|\mathcal{A}|})$ on $[0, T]$ for any $T>0$.

Now, the control operator is

$$
W_{u}: L^{2}(0, T) \ni u \rightarrow Y(T)=\sum_{n \in \mathbf{Z}}\left(b_{n}^{u}\left|\lambda_{n}\right|^{1 / 2} e^{\lambda_{n} t}, \tilde{v}\right) \Phi_{n} \in \mathcal{H}^{1 / 2}
$$


and the observation operator is

$$
\mathcal{O}_{u}: \mathcal{H}^{1 / 2} \ni Z_{0} \rightarrow w(t)=B_{u}^{*} Z_{0}(t)=\sum_{n \in \mathbf{Z}} z_{n} b_{n}^{u}\left|\lambda_{n}\right|^{1 / 2} e^{\lambda_{n} t} \in L^{2}(0, T) .
$$

In view of the Riesz basis property,

$$
\left\|W_{u} u\right\|_{H^{1 / 2}(0, T)} \asymp\|v\|_{L^{2}(0, T)}, \quad\left\|O_{u} Z_{0}\right\|_{L^{2}(0, T)} \asymp\left\|Z_{0}\right\|_{\mathcal{H}^{1 / 2}(0, T)} .
$$

Thus, we have obtained the following result.

Theorem 3.2. For any $T>0$, the system (25) with the control $u \in$ $L^{2}(0, T)$ is exactly controllable in the Sobolev space $\mathcal{H}^{1 / 2}(0, T)$; the system (26), with the observation $B_{u}^{*} Z=Z_{3} \in H^{1 / 2}(0, T)$, is exactly observable in the energy space $H$ on $[0, T]$.

Finally, we can find the estimates of the control and observations (18) and (19). Actually, in a way parallel to (19), we have

$$
C^{-1}(T)|n| \leq\left\|u_{n}\right\|_{L^{2}(0, T)} \leq C(T)|n|, \quad n \in \mathbf{Z},
$$

where the constant $C(T)$ depends on only $T$ and $u_{n}$ is the control with minimal norm that steers the rest initial state of the system (25) to $\Phi_{n}$.

\section{References}

1. Balakrishnan, A. V., and Taylor, L., The SCOLE Design Challenge, 3rd Annual NASA-SCOLE Workshop, NASA Technical Memorandum 89075, pp. 385-412, 1986.

2. Littman, W., and Markus, L., Exact Boundary Controllability of a Hybrid System of Elasticity, Archive for Rational Mechanics and Analysis, Vol. 103, pp. 193-235, 1988.

3. Guo, B. Z., On the Boundary Control of a Hybrid System with Variable Coefficients, Journal of Optimization Theory and Applications, Vol. 114, pp. 373-395, 2002.

4. RaO, B., Exact Boundary Controllability of a Hybrid System of Elasticity by the HUM Method, ESAIM, Control Optimization and Calculus Variations, Vol. 6, pp. 183-199, 2001.

5. Littman, W., and Markus, L., Stabilization of a Hybrid System of Elasticity be Feedback Boundary Damping, Annali di Matematica Pura ed Applicata, Vol. 152, pp. 281-330, 1988.

6. Rao, B., Uniform Stabilization of a Hybrid System of Elasticity, SIAM Journal on Control and Optimization, Vol. 33, pp. 440-454, 1995. 
7. Avdonin, S. A., and Ivanov, S. A., Families of Exponentials: The Method of Moments in Controllability Problems for Distributed-Parameter Systems, Cambridge University Press, Cambridge, UK, 1995.

8. SeIP, K., On the Connection between Exponential Bases and Certain Related Sequences in $L^{2}(-\pi, \pi)$, Journal of Functional Analysis, Vol. 130, pp. 131160, 1995.

9. Russell, D. L., On Exponential Bases for the Sobolev Spaces over an Interval, Journal of Mathematical Analysis and Applications, Vol. 87, pp. 528-550, 1982.

10. Ivanov, S., and Kalton, N., Interpolation of Subspaces and Applications to Exponential Bases, St. Petersburg Mathematical Journal, Vol. 13 pp. 221-239, 2002.

11. Curtain, R. F., and Zwart, H. J., An Introduction to Infinite-Dimensional Linear Systems Theory, Springer Verlag, New York, NY, 1995. 